\title{
THE QUIET SOLAR ATMOSPHERE OBSERVED AND SIMULATED IN Na I $\mathrm{D}_{1}$
}

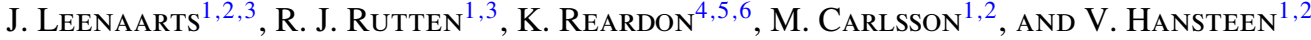 \\ ${ }^{1}$ Institute of Theoretical Astrophysics, University of Oslo, P.O. Box 1029, Blindern, N-0315 Oslo, Norway; jorritl@astro.uio.no \\ ${ }^{2}$ Center of Mathematics for Applications, University of Oslo, P.O. Box 1053, Blindern, N-0316 Oslo, Norway \\ ${ }^{3}$ Sterrekundig Instituut, Utrecht University, Postbus 80 000, NL-3508 TA, Utrecht, The Netherlands \\ ${ }^{4}$ INAF-Osservatorio Astrofisico di Arcetri, Largo Enrico Fermi 5, I-50125 Firenze, Italy \\ 5 Astrophysics Research Centre, Queen's University, Belfast, BT7 1NN, UK \\ ${ }^{6}$ NSO/Sacramento Peak, P.O. Box 62, Sunspot, NM 88349-0062, USA \\ Received 2009 May 10; accepted 2009 December 15; published 2010 January 13
}

\begin{abstract}
The $\mathrm{Na}$ I $\mathrm{D}_{1}$ line in the solar spectrum is sometimes attributed to the solar chromosphere. We study its formation in quiet-Sun network and internetwork. We first present high-resolution profile-resolved images taken in this line with the imaging spectrometer Interferometric Bidimensional Spectrometer at the Dunn Solar Telescope and compare these to simultaneous chromospheric images taken in Ca II $8542 \AA$ and $\mathrm{H} \alpha$. We then model $\mathrm{Na}$ I $\mathrm{D}_{1}$ formation by performing three-dimensional (3D) non-local thermodynamic equilibrium profile synthesis for a snapshot from a $3 \mathrm{D}$ radiation-magnetohydrodynamics simulation. We find that most $\mathrm{Na}_{\mathrm{I}} \mathrm{D}_{1}$ brightness is not chromospheric but samples the magnetic concentrations that make up the quiet-Sun network in the photosphere, well below the height where they merge into chromospheric canopies, with aureoles from 3D resonance scattering. The line core is sensitive to magneto-acoustic shocks in and near magnetic concentrations, where shocks occur deeper than elsewhere, and may provide evidence of heating deep within magnetic concentrations.
\end{abstract}

Key words: Sun: chromosphere - Sun: faculae, plages - Sun: magnetic topology - Sun: photosphere

\section{INTRODUCTION}

The past decade saw a rapid advance of our understanding of fine structure and dynamics in the lower atmosphere of the Sun by combination of high-resolution observations with increasingly realistic numerical simulations. Examples are the formation of the solar granulation (Nordlund et al. 2009) and reversed granulation (Leenaarts \& Wedemeyer-Böhm 2005; Cheung et al. 2007), and the magnetoconvective origin of intergranular bright points (e.g., Shelyag et al. 2004). Such studies focused mainly on photospheric structure, and assumed local thermodynamical equilibrium (LTE) in both the numerical simulation and the subsequent spectral-line synthesis.

The present frontier in this type of research is to study solar fine structure higher up in the solar atmosphere, including the chromosphere. There the magnetic field fans out and becomes more homogeneous, but shocks cause fine-scale structure and dynamics; LTE is no longer valid. Observationally, high-resolution and rapid-cadence imaging is required in spectral lines that sample these higher layers, with sufficient spectral resolution to chart and follow their small-scale and fast profile variations. At present only imaging Fabry-Pérot spectrometers at ground-based telescopes with adaptive optics perform this task adequately, sampling the few chromospheric lines in the red part of the visible spectrum.

In radiation-magnetohydrodynamics (MHD) simulations, such upward advance requires full three dimensionality and tackling the challenging complexities of non-LTE (NLTE) radiative transfer and non-equilibrium ionization/recombination balancing within the simulation. Post-processing in the form of subsequent synthesis of spectral diagnostics requires threedimensional (3D) NLTE radiative transfer, in some cases even with partial frequency distribution. In this paper, we make such an upward move for the quiet-Sun atmospheric regime sampled by the $\mathrm{Na}_{\mathrm{I}} \mathrm{D}_{1}$ resonance line at $5895.94 \AA$. This line is often taken to be chromospheric, but we show here that it is much less so than Ca II $8542 \AA$ or $\mathrm{H} \alpha$.
Recently, observational comparisons using Fabry-Pérot spectrometry were made between Ca II H \& K and Ca II 8542 A by Reardon et al. (2009) and between Ca II $8542 \AA$ and $\mathrm{H} \alpha$ by Cauzzi et al. (2009). Leenaarts et al. (2009) compared FabryPérot observations in Ca II $8542 \AA$ with numerical synthesis of this line from a 3D MHD simulation snapshot. The present paper is a companion to the latter study (hereinafter Paper I), using the same snapshot but turning to the $\mathrm{Na}_{\mathrm{I}} \mathrm{D}_{1}$ line. The major modeling advance in both papers is the incorporation of $3 \mathrm{D}$ NLTE radiative transfer for the profile synthesis of the respective line. Complete frequency re-distribution is assumed and is in order (Uitenbroek 1989; Uitenbroek \& Bruls 1992).

However, instantaneous LTE ionization equilibrium was assumed within the MHD simulation itself. Most importantly, it was assumed for hydrogen even though the studies by Carlsson \& Stein (2002) in one dimension (1D) and by Leenaarts et al. (2007) in two dimensions (2D) have shown that this is not an accurate assumption. Compared to LTE, hydrogen is underionized in hot shocks and overionized in cool post-shock conditions. Both are due to a large ionization timescale compared to the hydrodynamic evolution timescale. Improved treatment is highly desirable, but its implementation in 3D MHD simulation codes presents formidable challenges including severe processing-speed requirements. Paper I and the present paper therefore represent steps along the arduous obstacle course toward realistic ab initio simulation of the chromosphere, but not the final one.

In solar spectrum atlases, $\mathrm{Na} \mathrm{I}_{1}$ is about 5 times weaker than Ca II $8542 \AA$ in equivalent width, but its line center reaches deeper, about $200 \mathrm{~K}$ lower in brightness temperature. Na I $\mathrm{D}_{1}$ and Ca II $8542 \AA$ indeed differ in various formation properties. $\mathrm{Na} \mathrm{D}_{1}$ is a strongly scattering resonance line from an easily ionized minority stage; Ca II $8542 \AA$ is an excited line of the dominant calcium ionization stage and has larger sourcefunction coupling to the local temperature. These differences make it worthwhile to employ and compare both lines. 

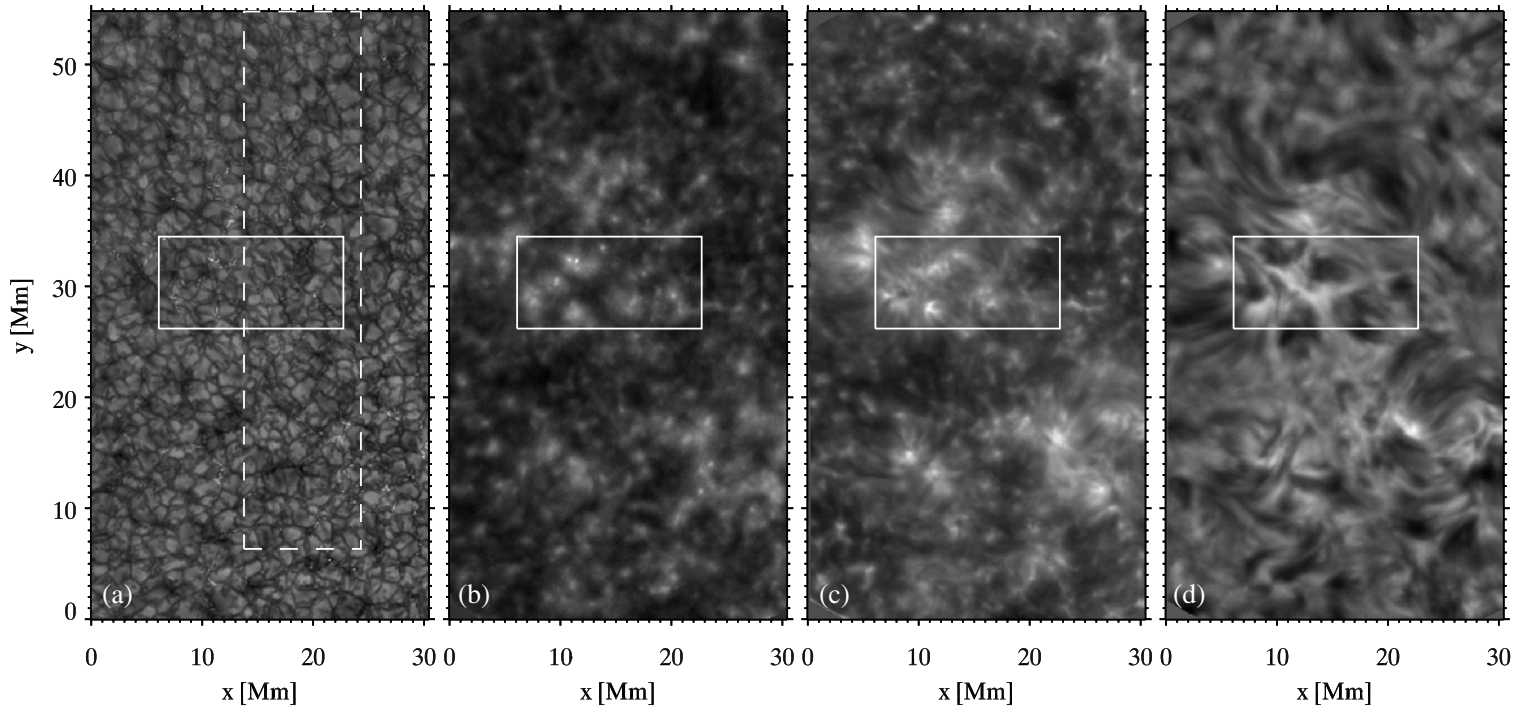

$$
\begin{array}{lll}
10 \quad 300 & 10 \\
\mathrm{x}[\mathrm{Mm}] & & \\
\mathrm{x}[\mathrm{Mm}]
\end{array}
$$
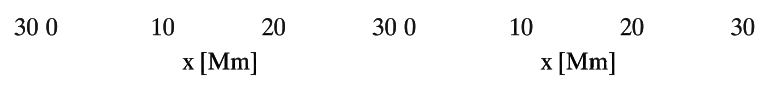

Figure 1. Images from the DST. Panels (a) G band, (b) Na I $D_{1}$, (c) Ca II $8542 \AA$, and (d) H $\alpha$. The latter three are constructed from the IBIS profile samplings by determining the intensity of the line profile minimum per pixel, in order to compensate for Dopplershifts from vertical bulk motions. The dashed box shows the overlap with the Hinode/SP magnetograms in Figure 2. The solid box is the subfield selected in Figure 3.

In the next section, we present and discuss high-resolution spectrally resolved imaging of a quiet-Sun area in Na I $D_{1}$. We then use 3D NLTE synthesis of this line in the MHD simulation snapshot of Paper I to analyze its formation in quietSun conditions (Section 3). We discuss the results in Section 4 and conclude the paper in Section 5.

\section{OBSERVATIONS}

\subsection{Data Acquisition}

We use imaging spectroscopy data obtained with the Interferometric Bidimensional Spectrometer (IBIS) at the Dunn Solar Telescope (DST) of the U.S. National Solar Observatory/ Sacramento Peak. IBIS consists of two Fabry-Pérot interferometers mounted in series and delivers sequences of narrowband images with the spectral passband stepping quickly through multiple user-selectable spectral lines (Cavallini 2006; Reardon \& Cavallini 2008). In this particular sequence, obtained during 15:30-17:07 UT on 2008 April 18, IBIS sampled Na I $D_{1}$ with a passband of $23 \mathrm{~m} \AA \mathrm{FWHM}$, Ca II $8542 \AA$ with a passband of $43 \mathrm{~m} \AA \mathrm{FWHM}, \mathrm{H} \alpha$ with a passband of $22 \mathrm{~m} \AA \mathrm{FWHM}$, and Fe I $7090 \AA$ with a passband of $24 \mathrm{~m} \AA \mathrm{FWHM}$. Additional images were taken in the $\mathrm{G}$ band with an independent camera using an interference filter of $10 \AA$ FWHM. One of the two adaptiveoptics feeds at the DST (Rimmele 2004) served to stabilize the telescopic image and to correct wavefront deformations in real time; the G-band images were additionally improved through speckle reconstruction. The $30 \times 54 \mathrm{Mm}^{2}$ field of view covered a quiet solar area near the edge of an equatorial coronal hole at apparent disk center (heliocentric longitude $0.56 \mathrm{~W}$, latitude $5.3 \mathrm{~S}$ ) with a pixel scale of $0.083 \mathrm{arcsec}_{\text {pixel }}{ }^{-1}$ (close to critically sampling the 0 '. $^{\prime} 16$ diffraction limit of the $76 \mathrm{~cm}$ telescope at $\mathrm{Na}$ I $\mathrm{D}_{1}$ ). As part of a service-mode observing program with Hinode a full-Stokes scan obtained with the Hinode/SP spectropolarimeter in the Fe I $6302.5 \AA$ line between 14:32 and 16:00 UT overlapped with a portion of the IBIS field of view.

\subsection{Results}

The IBIS images shown in Figures 1-3 were taken between 15:54:53 and 15:55:17 UT and were selected as the sharpest of

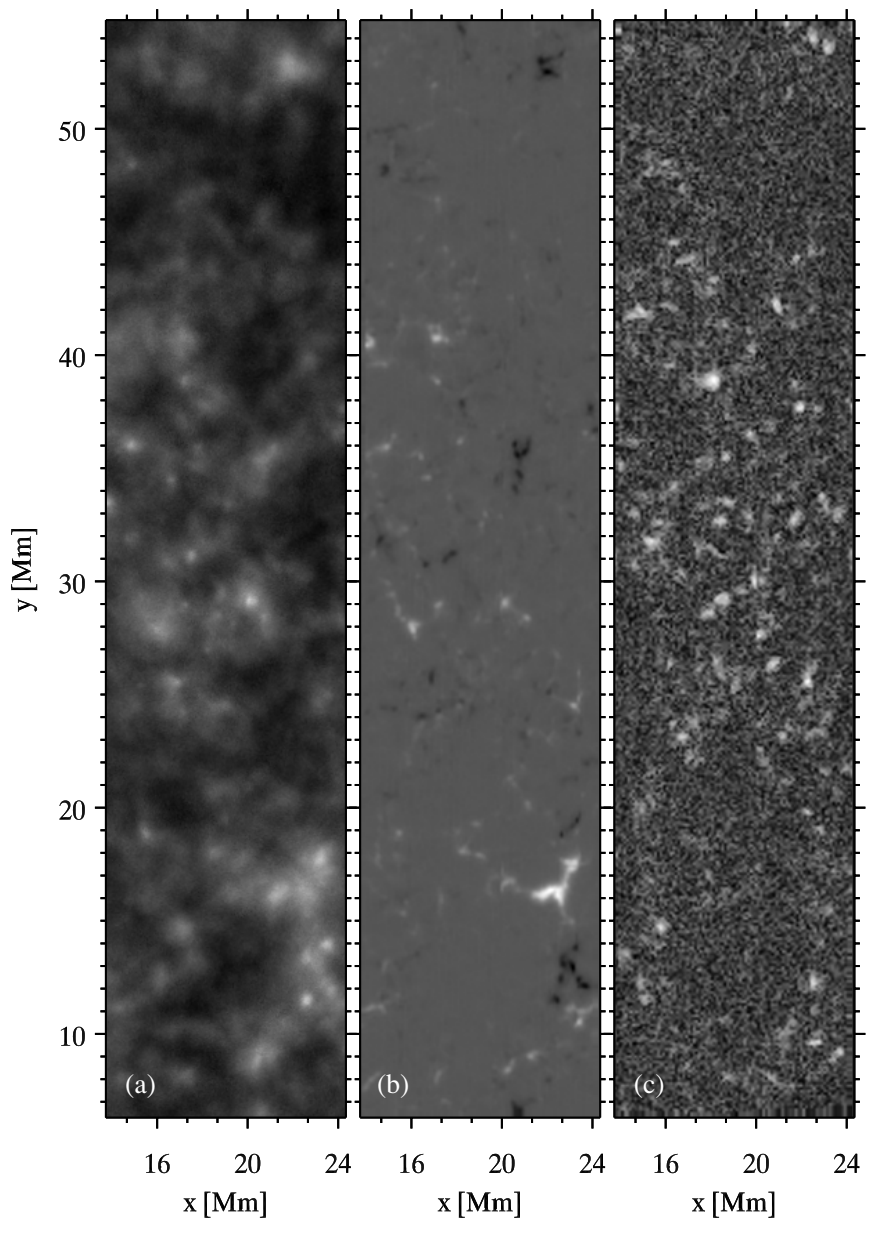

Figure 2. Na I $D_{1}$ profile-minimum intensity compared with longitudinal and transverse magnetograms for the overlap between the IBIS and Hinode/SP fields of view. Panel (a) the intensity of the Na I $D_{1}$ profile minimum per pixel, as in Figure 1; panel (b) apparent longitudinal flux density; panel (c) apparent transverse flux density. The flux density grayscale ranges from -441 to $+629 \mathrm{Mx} \mathrm{cm}^{-2}$ for panel (b) and 0 to $+338 \mathrm{Mx} \mathrm{cm}^{-2}$ for panel (c). The average measured flux density over the field of view is $70 \mathrm{Mx} \mathrm{cm}^{-2}$.

the sequence. The narrowband spectral images were additionally corrected by the application of destretching vectors derived from 

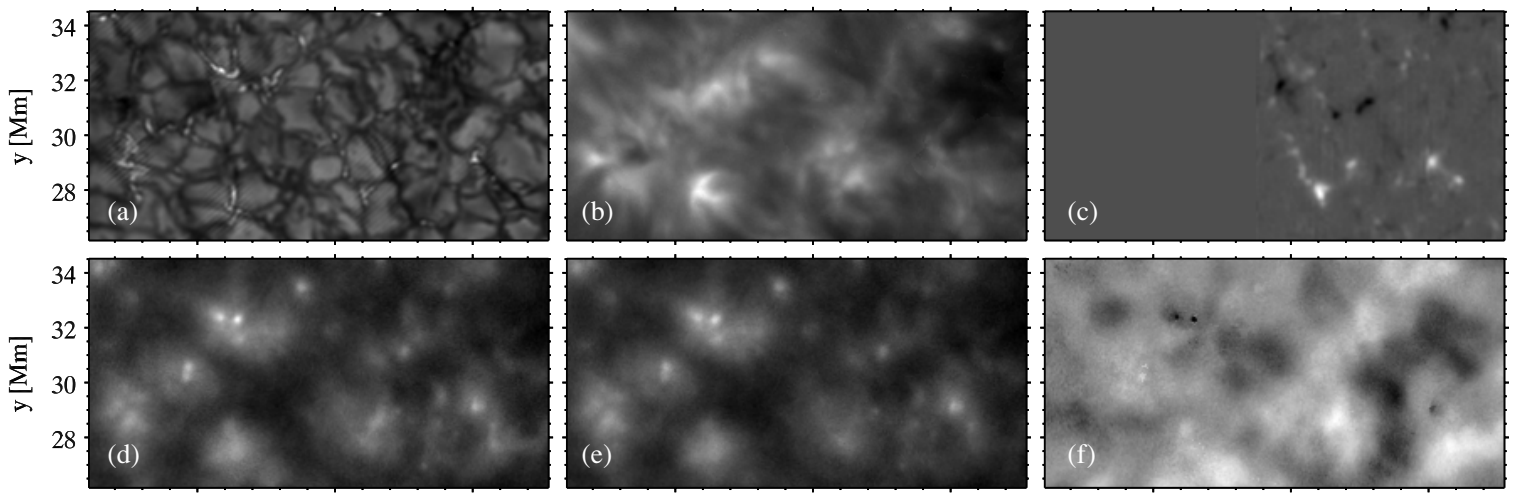

10

15

$\mathrm{x}$ [Mm]

20

\begin{abstract}
15
\end{abstract}
20

$\mathrm{x}$ [Mm]

10

15

$\mathrm{x}$ [Mm]

20

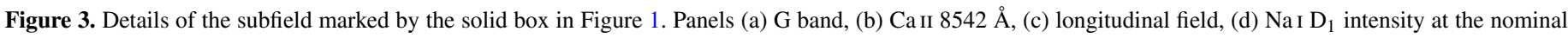

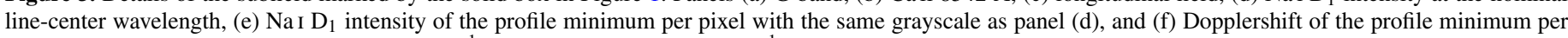
pixel, with the grayscale ranging from $-2 \mathrm{~km} \mathrm{~s}^{-1}$ (dark, downflow) to $+2 \mathrm{~km} \mathrm{~s}^{-1}$ (bright, upflow).

simultaneous broadband reference images. The dashed box in Figure 1 specifies the overlap with the Hinode/SP Stokes scan shown in Figure 2. The solid box outlines the subfield selected for Figure 3, chosen because it covers a similar area as our simulation with a comparable amount of magnetic network, including the most prominent bright points marking magnetic concentrations. However, only its right-hand half overlaps with the Hinode/SP scan.

The four images in Figure 1 differ clearly in their portrayal of the solar atmosphere, ranging from the photospheric Gband image at left to the chromospheric $\mathrm{H} \alpha$ image at right. These two extremes display characteristic quiet-Sun scenes. The G-band image shows regular deep-photosphere granulation with relatively few bright points marking strong-field magnetic concentrations, such as the network patch within the solid box. The $\mathrm{H} \alpha$ image shows chromospheric fibrils (or alternatively "mottles," but we use the term "fibril" for any elongated $\mathrm{H} \alpha$ structure), shorter and more irregularly shaped than would be the case in regions with larger magnetic activity. The fibrilar structure is more pronounced around the photospheric network.

The two intermediate images differ significantly from both the outer ones and also between each other. The Ca II $8542 \AA$ image shows some of the same fibrils seen in $\mathrm{H} \alpha$ around denser network, but in the quiet internetwork it shows a dark background with small roundish brightness patches that are mostly due to acoustic shocks (cf. Cauzzi et al. 2009). This regime was called "clapotisphere" by Rutten (1995), denoting sub-canopy domains with sufficiently weak field that acoustic and gravity waves can run up, become shocks, and interfere in intricate patterns. Similar patterns occur in fieldfree hydrodynamical simulations (Wedemeyer et al. 2004). The $\mathrm{Na}$ I $\mathrm{D}_{1}$ image (second panel) shows essentially no fibrilar structure, with only occasional glimpses of short and bright elongated structures. The brightest G-band bright points show up as co-spatial bright-point features, but with wide bright aureoles around them. The dark internetwork areas show vague pattern similarities with the Ca II $8542 \AA$ image. Thus, at first sight $\mathrm{Na}$ I $\mathrm{D}_{1}$ seems to sample the same internetwork waves, and possibly clapotispheric shocks, with photospheric magnetic concentrations visible as bright points with aureoles. The same is shown in the similar but lower-resolution images of $\mathrm{Al}$ et al. (2002).

With the lack of fibrils and only a few strong bright points in this quiet-Sun region, the $\mathrm{NaI}_{\mathrm{I}} \mathrm{D}_{1}$ image appears rather featureless. This apparent blandness is a solar property, since the broadband images taken simultaneously with the spectral images show excellent sharpness, especially near the adaptiveoptics lock point (located in the subfield shown in Figure 3). Righini et al. (2010) have shown that IBIS achieves near diffraction-limited image quality in its narrowband channel. Only small-scale features with very low contrast might drown in the photon noise set by the average line-core intensity of 2000 photons ixel $^{-1}$ during the $50 \mathrm{~ms}$ exposures. The variation in the appearance of the bright aureoles among different bright points and at different wavelengths shows that they do not result from scattering in the instrument or the Earth's atmosphere.

Figure 2 confirms the identification of $\mathrm{Na}$ I $\mathrm{D}_{1}$ bright points as magnetic concentrations. This part of the Hinode/SP Stokes scan lasted from 15:51:23 until 15:59:38 UT, so that it is not fully co-temporal with the $\mathrm{Na}_{\mathrm{I}} \mathrm{D}_{1}$ images. Nevertheless, there is remarkable correspondence between the brightest $\mathrm{Na}$ I $\mathrm{D}_{1}$ features and strong longitudinal field, much more than with the (generally much weaker) transversal field.

Figure 3 contains enlargements of the selected subfield. The magnetogram in panel (c) shows the signed longitudinal flux density but has only partial overlap. Panel (d) adds a filtergram display of the $\mathrm{NaI} \mathrm{D}_{1}$ intensity, i.e., with the IBIS passband at the nominal rest wavelength of the line, as compared to the image generated using local Doppler compensation in panel (e). The two panels have the same grayscale. The brightest features are brighter in panel (d) due to large central downflows which can be seen in panel (f) as three tiny very dark patches in the middle of magnetic concentrations. Three other magnetic concentrations display comparably narrow updrafts. These motions Doppler-shift the line core out of the passband, causing additional brightening, as also pointed out by Al et al. (2002). Such mixing of Dopplershift and intensity modulation generally affects standard fixed-wavelength narrowband filtergrams. We therefore plot Doppler-compensated line-center intensities rather than rest-wavelength line-center intensities, so that brightness variations are more indicative of the source function than of the velocity field in the line forming region. The larger-scale patterning in panel (f) has no obvious correspondence to the other panels.

The upshot of this section is that the Na I D1 core brightness seems to coincide more with the photospheric Stokes- $V$ magnetogram signal than with fibrilar structure seen in Ca II $8542 \AA$ and $\mathrm{H} \alpha$. This suggestion agrees with the earlier conclusion of 
Cauzzi et al. (2000) from lower-resolution (spatial and spectral) data that the $\mathrm{NaI} \mathrm{D}_{2}$ core intensity correlates well with the apparent magnetic flux density in network (above $50 \mathrm{Mx} \mathrm{cm}^{-2}$ in their Figure A1). Figure 1 indicates that $\mathrm{Na}_{\mathrm{I}} \mathrm{D}_{1}$ in the internetwork may show some clapotispheric intensity modulation caused by waves and shocks, mimicking those in Ca II $8542 \AA$, but none of the chromospheric structure seen in $\mathrm{H} \alpha$.

\section{SIMULATIONS}

\subsection{MHD Simulation Snapshot}

We used a snapshot from a radiation-MHD simulation computed with the Oslo Stagger Code (OSC; Hansteen et al. 2007). The same snapshot was used in Paper I. OSC includes an LTE equation of state based on the Uppsala Opacity Package (Gustafsson 1973) and uses radiative transfer with multigroup opacity binning following Nordlund (1982) and NLTE scattering following Skartlien (2000) to compute radiative losses in the photosphere and low chromosphere. Radiative losses in the middle and upper chromosphere are evaluated with an escape probability method including continua and lines of hydrogen plus lines of Ca II. It was calibrated with the 1D RADYN code (e.g., Carlsson \& Stein 1997). Higher up, OSC employs optically thin radiative cooling. Thermal conduction along magnetic field lines is taken into account. More detail is given in Hansteen et al. (2007) and Martínez-Sykora et al. (2008, 2009). The simulation used here has a grid of $256 \times 128 \times 160$, corresponding to a physical size of $16.6 \times 8.3 \times 15.5 \mathrm{Mm}^{3}$. It extends from $1.5 \mathrm{Mm}$ below the photosphere to $14 \mathrm{Mm}$ above it, i.e., from the upper convection zone into the corona. The grid has $65 \mathrm{~km}$ horizontal resolution. The grid is non-uniform in the vertical direction; its resolution varies from $32 \mathrm{~km}$ in the convection zone to $440 \mathrm{~km}$ in the corona. The snapshot contains bipolar magnetic field with a mean strength of $150 \mathrm{G}$ in the photosphere.

\subsection{Radiative Transfer}

For the radiative transfer computation of the emergent $\mathrm{Na}_{\mathrm{I}} \mathrm{D}_{1}$ intensity, the atmosphere was interpolated in the vertical direction onto a grid with 153 points covering a height range between -0.5 and $2 \mathrm{Mm}$ relative to continuum optical depth $\tau_{5000}=1$, covering the formation range of all pertinent sodium transitions. The electron density was computed assuming LTE ionization for all relevant species, including hydrogen, consistent with the LTE equation-of-state used in OSC. The angle quadrature was computed using the A4 set of Carlson (1963), employing three rays per octant. The gas motions in the simulation were taken into account without adding additional microturbulence.

The radiative transfer computation was performed using MULTI3D (Botnen 1997; Leenaarts \& Carlsson 2010), a code based on the 1D code MULTI of Carlsson (1986) and including the same physics, but with a 3D short-characteristics solver allowing evaluation of the 3D radiation field. MULTI3D has been $\mathrm{MPI}^{7}$-parallelized using domain decomposition so that it runs efficiently on supercomputers.

Since 3D radiative transfer remains very demanding, the actual Na I Grotrian diagram was simplified to a minimal model atom. Inspired by the results of Bruls et al. (1992), we constructed a model atom of only four levels, consisting of the ground state, the upper level of $\mathrm{Na}$ I $\mathrm{D}_{1}$, an extra level at $1 \mathrm{eV}$ below the continuum, and the Na II ground state as continuum. We gave the extra level normal collisional coupling to

\footnotetext{
Message Passing Interface.
}
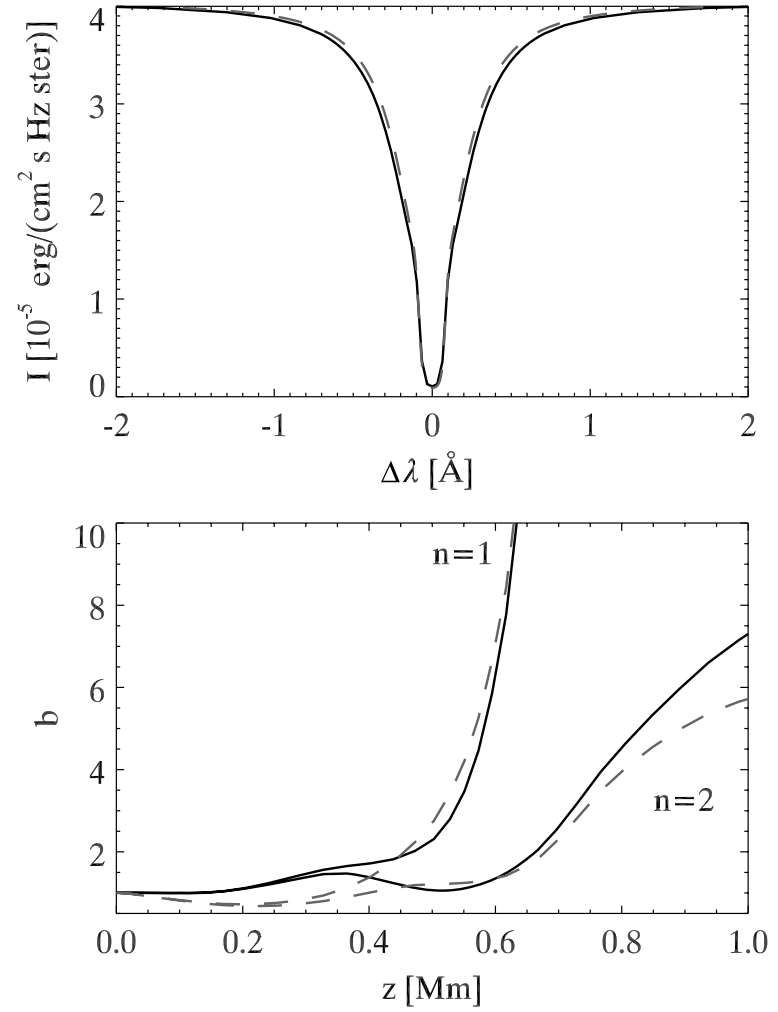

Figure 4. Comparison of Na I $D_{1}$ line formation in the FALC atmosphere between the 18-level (black curves) and 4-level (gray dashed curves) model atoms. Upper panel: emergent line profiles at $\mu=0.95$. Lower panel: NLTE population departure coefficients for the lower $(n=1)$ and upper $(n=2)$ level of the line.

the two lower levels but enormous $\left(10^{5}\right.$ in excess of normal values) collisional bound-free coupling to the continuum and large (Gaunt factor 2.2) radiative coupling to the upper level of Na I $\mathrm{D}_{1}$. We added this extra level to implement the suction process identified by Bruls et al. (1992) which draws population from the ion reservoir into the Na I D ground state. We adjusted its strong couplings such that it has similar suction power as the complete full-atom upper term structure, which we determined by comparing the resulting $\mathrm{Na}$ I $\mathrm{D}_{1}$ level populations to those of the full 18-level model atom specified in Table 2 of Bruls et al. (1992) for the standard 1D FALC atmosphere of Fontenla et al. (1993). The result is shown in Figure 4. The emergent profiles in the upper panel are nearly equal. The lower panel compares the NLTE population departure coefficients $b_{\mathrm{i}} \equiv n_{\mathrm{i}}^{\mathrm{NLTE}} / n_{\mathrm{i}}^{\mathrm{LTE}}$ for the lower and upper Na I $\mathrm{D}_{1}$ level between the two model atoms. They match very well, especially over the most important range $z=0.4-0.6 \mathrm{Mm}$ where the line core becomes optically thin. The steep increase of the $b_{1}$ departure coefficient is due to the dominance of photoionization over collisional ionization, but sufficient suction is needed to match it with the small atom. Slightly larger differences occur deeper down, where both small-atom curves miss the slight overpopulation in the FALC temperature minimum due to lack of photon suction in weaker lines. However, the ratio $b_{2} / b_{1}$ remains nearly equal between the two models so that the $\mathrm{Na}$ I $\mathrm{D}_{1}$ source functions are nearly equal; only the optical-depth buildup below the formation height of the line core differs somewhat. This simple model atom is accurate enough for our purposes. We used it to compute emergent $\mathrm{Na}$ I $\mathrm{D}_{1}$ profiles from the simulation snapshot, both treating each column as a plane-parallel 

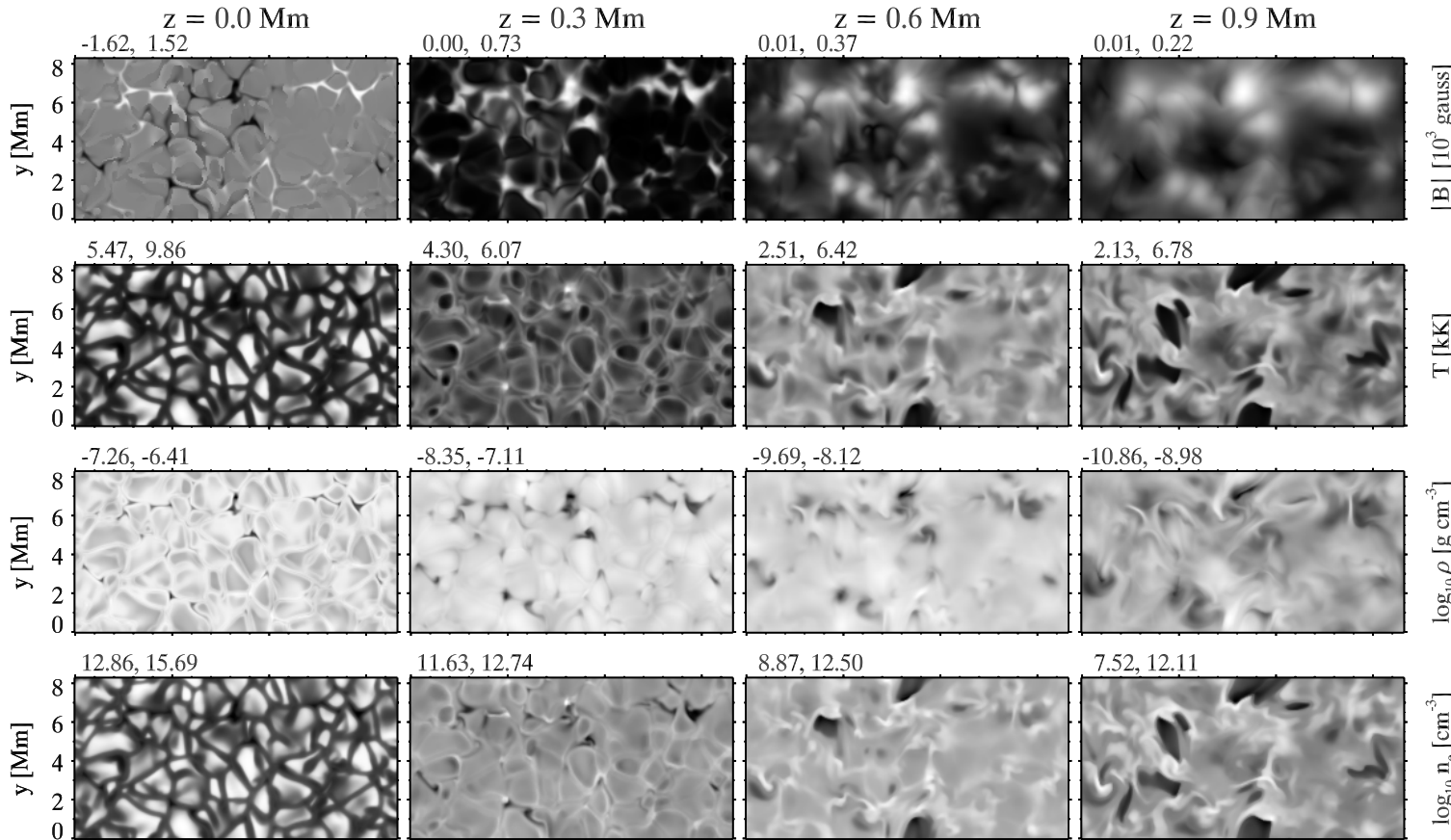

$\frac{\sqrt{2}}{5}$
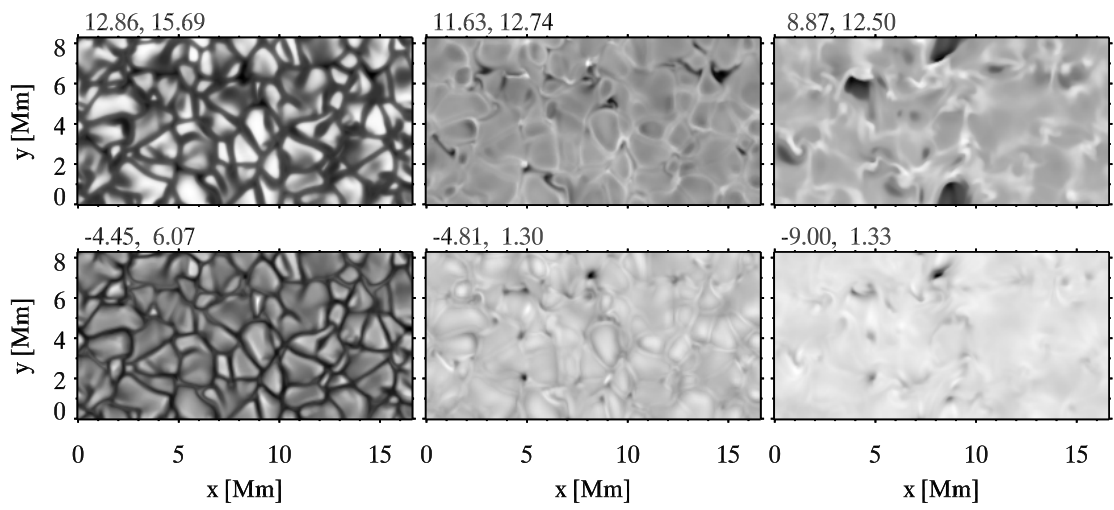

$-10.86,-8.98$
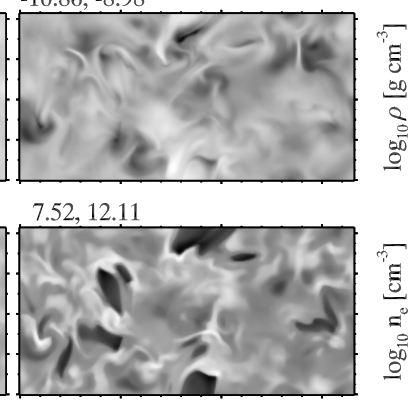

$-9.00,1.33$

$-12.65,2.85$
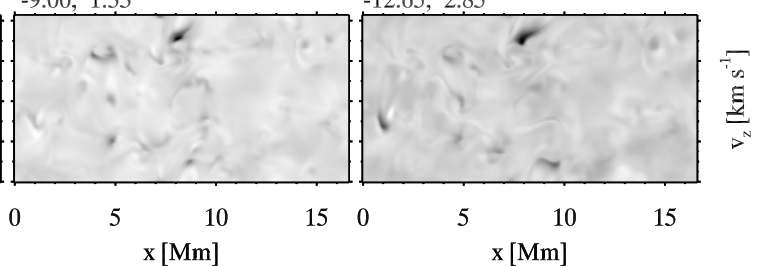

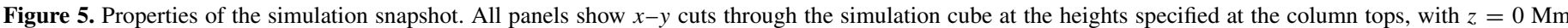

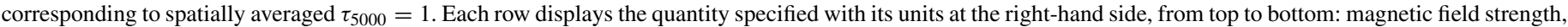

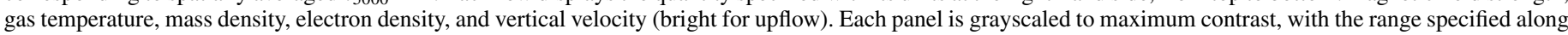
each panel top. The top-left panel displays the magnetic field strength multiplied with the sign of $B_{\mathrm{Z}}$ (upward bright) to show the polarity distribution.

atmosphere and in full 3D, assuming complete redistribution which is a good assumption for this line (Uitenbroek \& Bruls 1992).

\subsection{Snapshot Properties}

Figure 5 displays simulation results in the form of horizontal cuts through the snapshot cube at four different heights, specified at the top of each column.

The first row shows how the magnetic field is organized in small-scale intergranular magnetic concentrations, especially at lane vertices, that expand with height as in the classical fluted flux tube paradigm (e.g., Spruit 1977; Solanki 1987, 1993). At $z=0.0 \mathrm{Mm}$ (upper left panel) they do not appear as roundish flux tubes but rather as extended features similar to the G-band "ribbons" and "flowers" in observations with comparable resolution (Berger et al. 2004; Rouppe van der Voort et al. 2005). The expansion with height is striking (note the scale reduction to 7 times lower amplitude at $z=0.9 \mathrm{Mm}$ ). The actual field is bipolar with a roughly even split between positive and negative concentrations, as shown in the first panel (where the field is mostly near-vertical).

The temperature cut at $z=0.0 \mathrm{Mm}$ in the second row shows granulation. At $z=0.3 \mathrm{Mm}$ the brightness pattern has changed into reversed granulation; the strongest magnetic concentrations stand out by being hotter than their surroundings at equal geometrical height. Higher up, fairly large dark patches ("clouds") of very cool gas occur, with hot white borders indicative of shocks. They occur preferentially near magnetic polarity changes.

The gas density (third row) shows the familiar evacuation of magnetic concentrations. At $z=0.0 \mathrm{Mm}$ these are superimposed on the higher-density intergranular lanes that appear as negative of the temperature pattern. At $z=0.3 \mathrm{Mm}$ the tops of the largest granules are densest. The magnetic evacuation persists at all sampled heights, spreading with the field strength but maintaining co-spatiality with the photospheric topology up to $z=0.6 \mathrm{Mm}$. In the $z=0.9 \mathrm{Mm}$ panel, the gas density shows slender high-density features that likely mark shock interference.

The electron density (fourth row) mimics the temperature closely at all heights because both the hydrogen ionization balance and the electron-donor $(\mathrm{Si}, \mathrm{Fe}, \mathrm{Al}, \mathrm{Mg}, \mathrm{Ca}, \mathrm{Na}$ ) ionization balances are computed in LTE. At $z=0.3 \mathrm{Mm}$ most magnetic concentrations have low electron density due to low overall gas density, but the hottest show excess electron density from donor ionization. The $N_{e} / N_{\mathrm{H}}$ ratio of electron to hydrogen density (not shown) reaches values as low as $10^{-6}$ in the coolest clouds in the higher layers, well below the $10^{-4}$ relative abundance of the electron-donor elements, implying that even these are largely neutral. The thin high-temperature shocks around the cool clouds have large electron densities.

The vertical velocity pattern in the bottom row shows the familiar pattern of granular upflow and downflow in the 


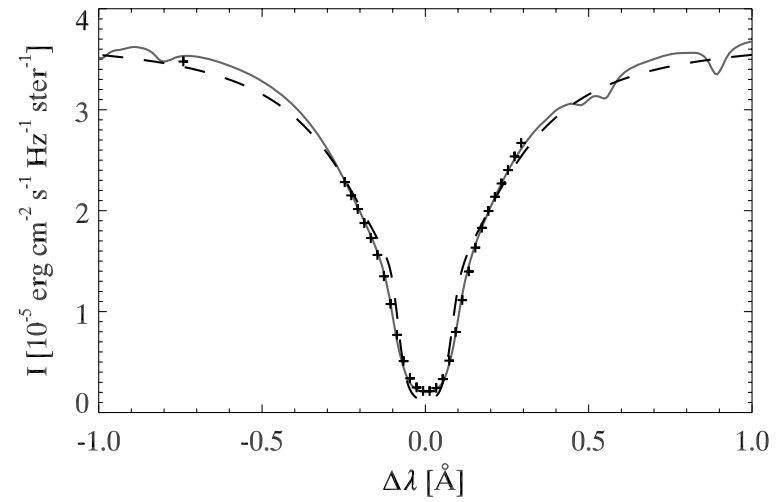

Figure 6. Comparison of spatially averaged observed and simulated $\mathrm{Na}_{\mathrm{I}} \mathrm{D}_{1}$ line profiles. Solid gray curve: averaged disk-center intensity from the NSO Fourier transform spectrometer (FTS) atlas calibrated by Neckel \& Labs (1984). Plus signs: IBIS observation. Dashed curve: simulation. Both the FTS atlas and the simulation provide absolute intensities. The IBIS data were scaled to match the FTS profile in the line core.

photosphere. Its amplitude diminishes rapidly with height. Higher up, the most striking features are large downdrafts in most of the stronger magnetic concentrations, in particular, the strongest one at $(x, y)=(8.2,7.2)$. They survive up to $z=0.9 \mathrm{Mm}$. The upflow patterns resemble the gas density patterns.

\subsection{Emergent Na I $D_{1}$ Profiles}

Figure 6 compares the spatially averaged $\mathrm{Na}$ I $\mathrm{D}_{1}$ profile from the simulation with the spatially averaged IBIS observation and with a solar-atlas profile. The agreement between the IBIS and atlas profiles is excellent. The simulation deviates in producing a slightly deeper core and narrower inner wings, with more pronounced knees. This lack of core broadening is similar to, but smaller than for the Ca II $8542 \AA$ line in Figure 1 of Paper I. The latter was attributed, at least partially, to a lack of spatial resolution in the microturbulence-free simulation. Since we use the same snapshot, this deficiency also applies here. However, its effect is probably smaller because $\mathrm{Na}_{\mathrm{I}} \mathrm{D}_{1}$ is formed lower than Ca II $8542 \AA$ whereas the temperature and density in Figure 5 show an increase in fine-structure amplitude with height.

Figure 7 displays properties of the $\mathrm{NaI} \mathrm{D}_{1}$ spectral profiles that emerge vertically from the top of the simulation volume, in the form of images comparable to the lower row in Figure 3. The wing image in the first panel serves as low-photosphere indicator, showing the magnetic concentrations as bright points. The blue $\mathrm{Na}$ I $\mathrm{D}_{1}$ wing was not included in our earlier survey of proxy-magnetometry diagnostics (Leenaarts et al. 2006), but it is comparable to the other strong-line wings there. The magnetic concentrations gain contrast because of weaker damping wings, and, in the case of $\mathrm{Na}_{\mathrm{I}} \mathrm{D}_{1}$ also because of enhanced ionization from partial evacuation.

The second panel of Figure 7 emulates a narrowband filtergram with the passband at nominal line center. The strongest magnetic concentration appears very bright, mostly through downdraft Dopplershift as demonstrated in Figure 9. The rest of the scene vanishes by setting the grayscale to include this feature.

Figures 7(c) and (d) compare 1D plane-parallel per-column radiative transfer with full $3 \mathrm{D}$ radiative transfer, plotting the profile-minimum intensity per pixel in order to compensate for bulk-motion Dopplershifts. The grayscales are identical. The scenes are comparable but the magnetic concentrations
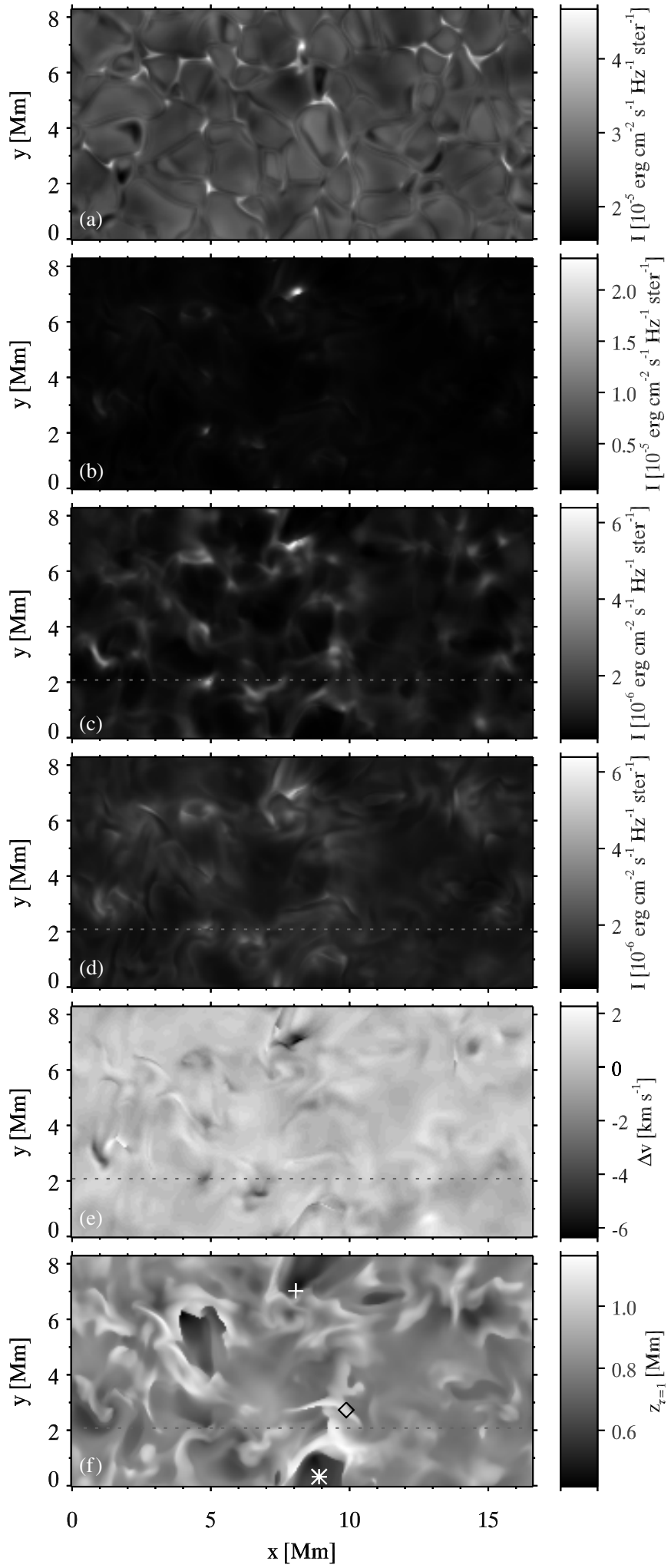

Figure 7. $\mathrm{Na} I \mathrm{D}_{1}$ synthesis with the $3 \mathrm{D}$ solver, except for panel (c) The intensity maps show the vertically emergent intensity from the simulation. From top to bottom: (a) intensity in the blue wing at $\Delta \lambda=$ $-0.27 \AA$ from nominal line center; (b) intensity at the nominal line center (rest wavelength); (c) intensity in the profile minimum per pixel, using the 1D solver; (d) intensity in the profile minimum per pixel; (e) Dopplershift of the profile minimum per pixel (upflows bright); (f) $\tau=1$ height for the profile minimum per pixel. The dotted line near $y=2.0 \mathrm{Mm}$ specifies the cut location used in Figure 8 . The three symbols in the bottom panel specify the locations for which $\mathrm{Na} \mathrm{I}_{1}$ formation breakdown is given in Figure 9. 

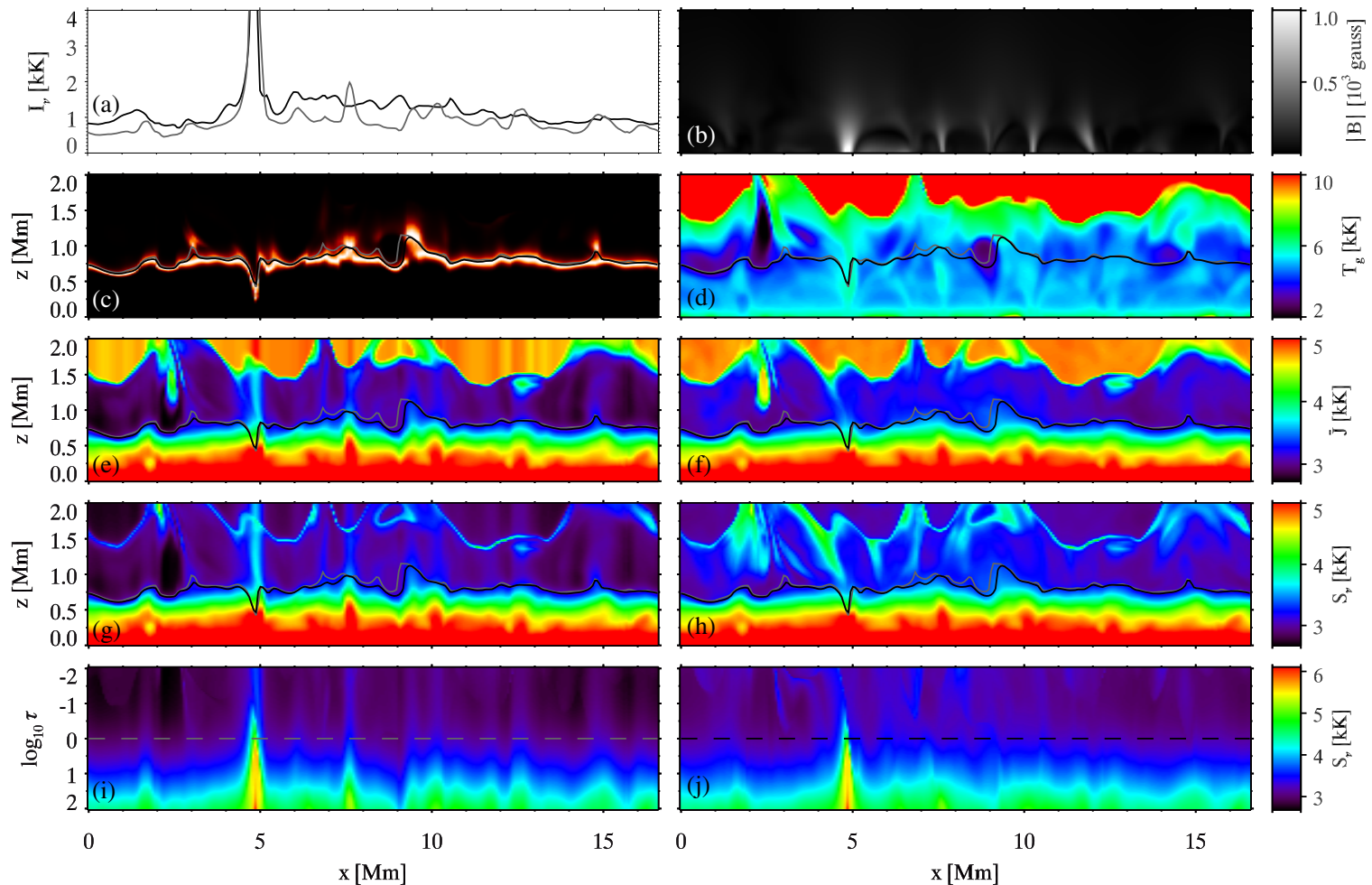

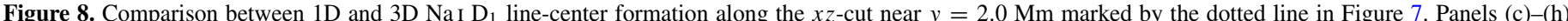

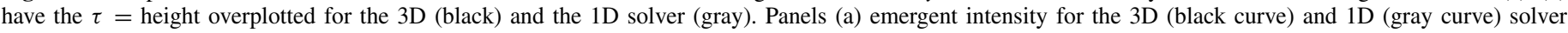

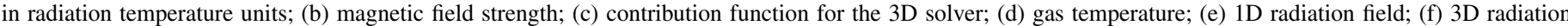

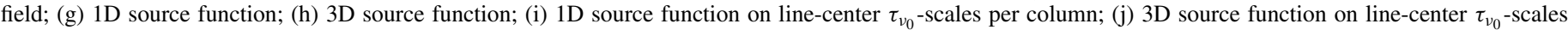
per column.

reach larger contrast in the $1 \mathrm{D}$ case. In $3 \mathrm{D}$, radiation scatters out of these and illuminates their surroundings, as shown below.

Figure 7(e) maps the Dopplershift of the line profile minimum per pixel. The grayish small-amplitude bright and dark patterning resembles the $z=0.9 \mathrm{Mm}$ vertical-velocity sampling in the last panel of Figure 5. The larger magnetic-concentration downdrafts mimic those in the $z=0.6 \mathrm{Mm}$ panel most closely. Narrow bright structures due to upward-propagating shocks appear elsewhere.

Finely structured bright shocks and dark clouds appear abundantly in Figure 7(f), which displays the geometrical height where radiation at the wavelength of the profile minimum per pixel reaches optical depth unity. The range is very large, varying from $z=0.5 \mathrm{Mm}$ in the cool clouds to $z=1.1 \mathrm{Mm}$ in the shock fronts. This rugged height-of-formation pattern is effectively a map of the $\mathrm{Na}$ I/Na II ionization-recombination balance and is closely similar to the electron density pattern at $z=0.9 \mathrm{Mm}$ in Figure 5, adding the slender high-density features in the panel above it.

In the cool clouds, sodium is nearly completely ionized. This counterintuitive result (one would expect sodium to be largely neutral at these temperatures and densities) is explained as follows: the electron density in the model atmosphere is computed in LTE and the cool clouds have thus a low electron density. The NLTE sodium ionization-recombination balance is set by the interplay of photoionization versus photorecombination requiring one electron. The photoionization rate per sodium atom is independent of temperature, as it is set by the radiation field. The recombination rate per sodium atom however depends on the electron density. In the low-temperature clouds, the electron density is low, thus, the recombination rate is low and as a consequence the ionization-recombination balance shifts toward Na II.

The Na I $D_{1} \tau=1$ height map obtains its enormous variation and fine structure from the large variations in electron density affecting the sodium recombination. The Dopplershift map in Figure 7(e) follows this $\tau=1$ height variation; it samples the Dopplershift in magnetic concentrations and cool clouds deeper in the atmosphere than in hot shocks.

Note that the $\tau=1$ height variation would be even larger if we had not plotted $\tau_{v}=1$ for the Doppler-compensated profile minimum per pixel but $\tau_{\nu_{0}}=1$ at the nominal line center as in a narrowband filtergram. In that case Dopplershifts would cause even deeper line-center formation, as deep as $z=0.3 \mathrm{Mm}$ in the downdrafts in the magnetic concentrations (Figure 9).

\subsection{Comparison of $3 D$ and $1 D$ Modeling}

Figure 8 compares our $3 \mathrm{D}$ evaluation of $\mathrm{Na} \mathrm{I}_{1}$ formation to the $1 \mathrm{D}$ one by showing an $x z$-cut through the simulation volume along the $y=2.0 \mathrm{Mm}$ dotted line in Figure 7 where it passes through the second-brightest magnetic concentration in panels (b) and (c). The magnetic concentration stands out in panel (b) of Figure 8, which also illustrates its upward field spreading, and in panel (d) as a relatively hot deep finger. The cut passes through a few more intergranular lanes with fairly strong field but no vertex concentration and samples a high cool cloud at $x=2.5 \mathrm{Mm}$ and a low one at $x=8.9 \mathrm{Mm}$. 

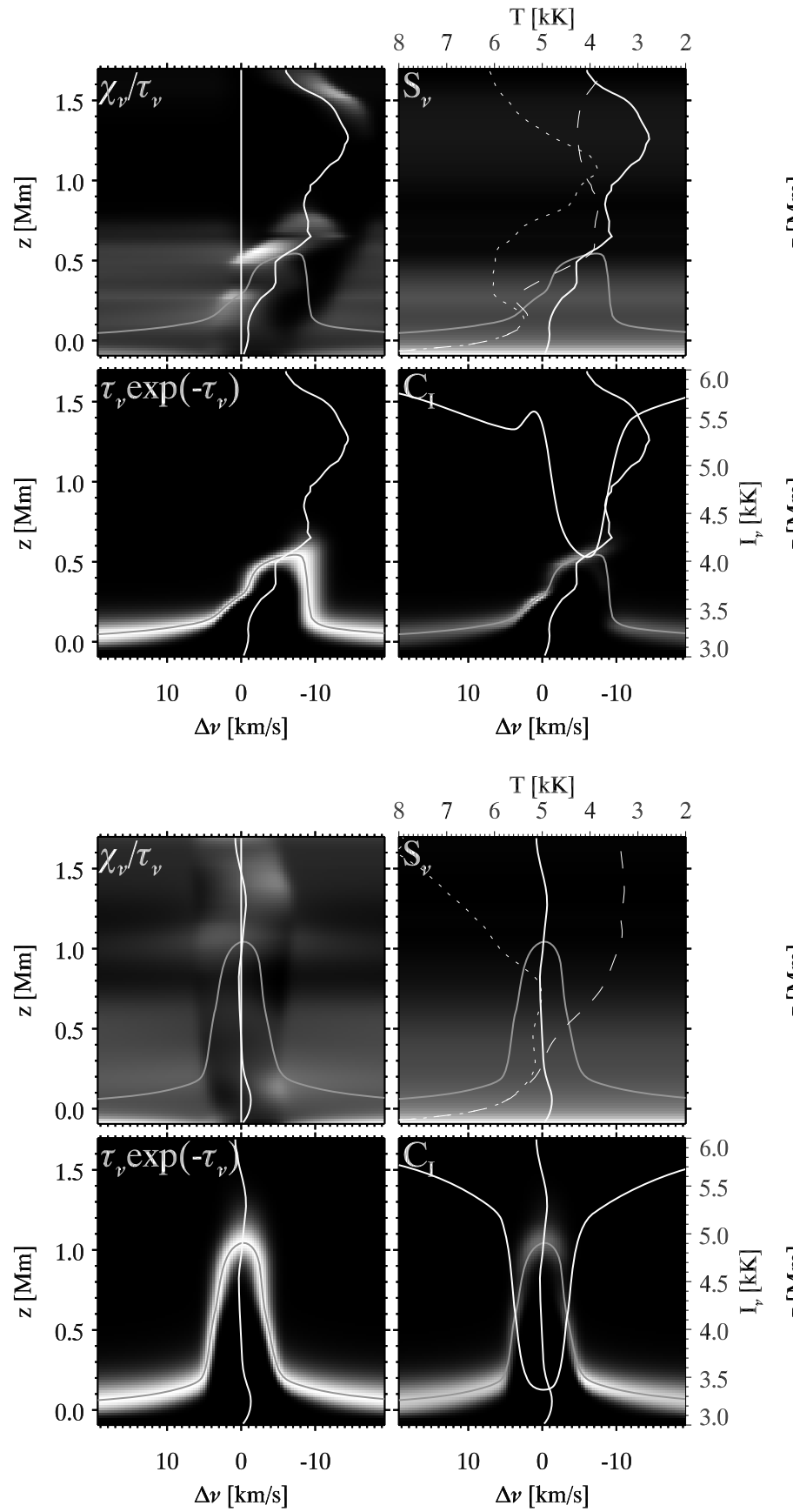
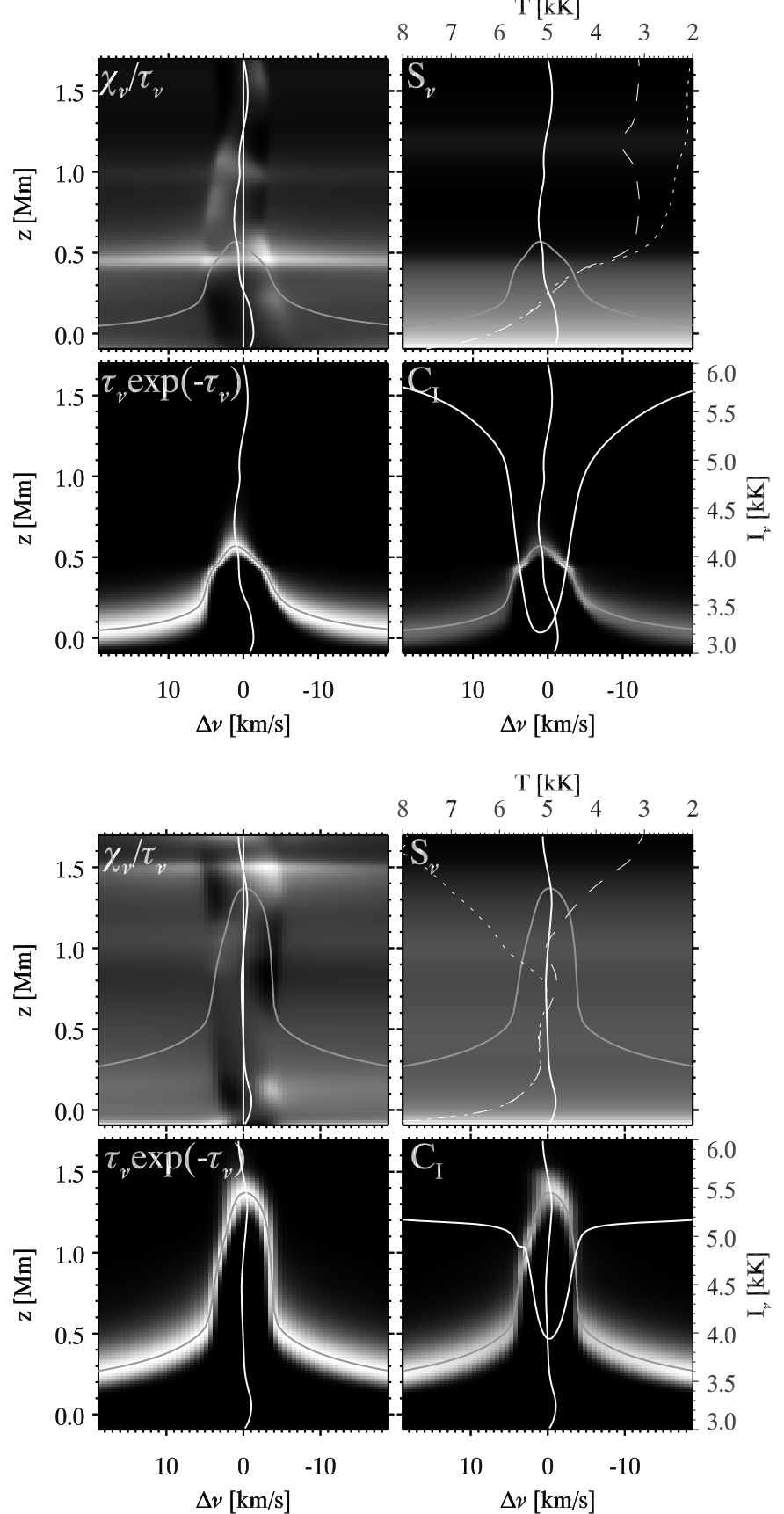

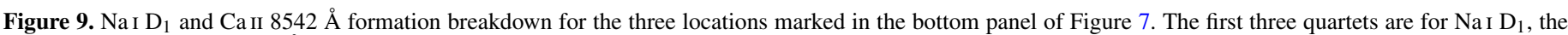

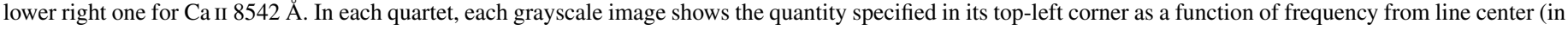

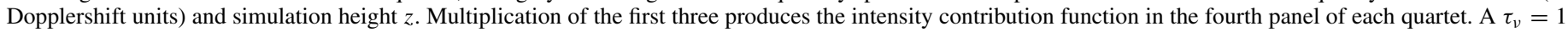

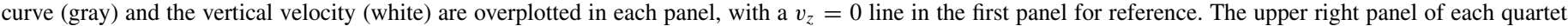

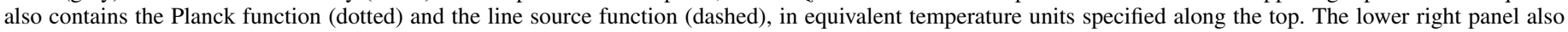

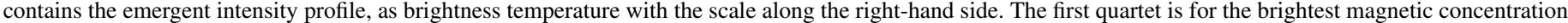

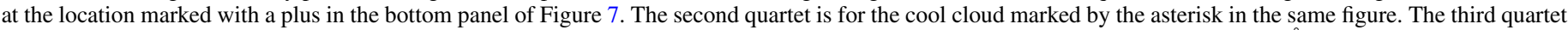
is for the hot front marked by the rhombus. The final quartet is for the same location but shows the formation breakdown for Ca II $8542 \AA$.

The first panel shows the vertically emergent intensity at the nominal line-center wavelength along the cut, given in equivalent brightness-temperature units to facilitate comparison with Figure 8(d)-(j). The 3D curve has an aureole around the magnetic concentration and is generally much brighter and smoother than the 1D curve. Panel (c) displays the 3D vertical contribution function in color. The nominal line-center $\tau_{\nu_{0}}=1$ heights are overplotted, also in panels (d)-(h) as reference; they do not differ much between 3D and 1D. The line-center formation height varies from $z \approx 0.4 \mathrm{Mm}$ in the magnetic concentration to $z \approx 1.1 \mathrm{Mm}$ in the hot rim of the deep cool cloud that appears as a filamentary feature in the $z=0.6 \mathrm{Mm}$ temperature panel of Figure 5. The other upward spikes in panel (c) sample similar features, all with very wide contribution functions. The cool clouds cause $\tau_{\nu_{0}}=1$ dips.

The lower three rows of Figure 8 analyze $\mathrm{Na}$ I $\mathrm{D}_{1}$ source function properties, at left for the $1 \mathrm{D}$ case and at right for the 3D case. Panels (e) and (f) map the profile-averaged angle-averaged 
radiation field

$$
\bar{J} \equiv \frac{1}{4 \pi} \int_{0}^{\infty} \int_{0}^{2 \pi} \int_{0}^{\pi} I_{\nu} \varphi\left(\nu-v_{0}\right) \sin \theta d \theta d \phi d \nu,
$$

with $I_{\nu}$ being the local specific intensity, $\varphi\left(v-v_{0}\right)$ the local $\mathrm{Na}$ I $\mathrm{D}_{1}$ area-normalized extinction profile, $v$ the frequency, $v_{0}$ the nominal line-center rest frequency, and $\theta$ and $\phi$ specify the viewing angle in spherical coordinates. The other panels map the total source function at $v=v_{0}$. It can be written, dropping frequency indices, as

$$
S=(1-\varepsilon)(1-\eta) \bar{J}+\varepsilon(1-\eta) B(T)+\eta(1-\varepsilon) B^{*},
$$

where $\bar{J}$ represents the $\mathrm{Na}_{\mathrm{I}} \mathrm{D}_{1}$ photon reservoir, $B(T)$ the thermal reservoir, and $B^{*}$ the reservoir for roundabout paths (in particular recombination cascades) that may populate the upper level and hence produce line photons. $B^{*}$ is only a formal Planck function, not necessarily corresponding to the local temperature. The coefficient $\varepsilon$ is the thermal line-photon destruction probability by collisional de-excitation after photoexcitation in the $\mathrm{Na}$ I $\mathrm{D}_{1}$ transition and through $\mathrm{H}^{-}$photoionization as background continuum process. The profile weighting with the latter contribution makes the total source function frequency dependent, even though the line source function is not for complete redistribution. The coefficient $\eta$ is the probability of line-photon conversion via other "interlocking" sodium transitions from the upper level (in particular photoionization).

Figures 8(e) and (f) are identical in deep layers where $\bar{J} \approx B$. Higher up, the two panels appear identical also, in this case because their structure is dominated by the thermal broadening in the $\varphi\left(v-v_{0}\right)$ profile weighting. Toward the corona (note that the red color in panel (d) represents the cutoff at $T=10^{4} \mathrm{~K}$, the actual temperatures are higher) the line profile becomes so wide that $\bar{J}$ approaches the emergent-continuum value with $T_{\text {rad }} \approx 5100 \mathrm{~K}$ (see the $500 \mathrm{~nm}$ panel of Figure 36 of Vernazza et al. 1981; or Figure 3 of Bruls et al. 1992). In the 1D case, this part (orange) is patterned as vertical search-light beams above hot granules. They are washed out in the 3D case. Similar washout occurs in the blue-black regime with $T_{\text {rad }}<3600 \mathrm{~K}$ where $\mathrm{Na}$ I $\mathrm{D}_{1}$ is formed; for example, the cooler areas above $\tau=1$ in panel (d) become black in panel (e) but are filled with warmer radiation in panel (f). The scattering contribution dominates the source function in panels $(\mathrm{g})$ and $(\mathrm{h})$ in the blue domain around $z=1.0 \mathrm{Mm}$, but higher up the $\varepsilon B(T)$ and $\eta B^{*}$ contributions mix in, the first producing the thin bluish-white curve marking the temperature rise to the corona (where $\varepsilon$ is negligible), the second producing the slanted greenish features below this curve. The $\varepsilon B(T)$ contribution is the same in 1D and 3D because it is local, but there is marked difference between $1 \mathrm{D}$ and $3 \mathrm{D}$ for the $\eta B^{*}$ contribution due to non-local ionizing ultraviolet radiation. However, these contributions are too high up to affect the $\mathrm{Na}$ I $\mathrm{D}_{1}$ brightness, as is demonstrated in panels (i) and (j) by plotting the source function per vertical column on the relevant part of the corresponding line-center $\tau_{v_{0}}$ scale. The magnetic concentration is the hottest feature crossing the $\tau_{\nu_{0}}=1$ line; all other locations sample the blue $\bar{J}$-dominated scattering regime, of which the columnar structure in 1D explains the modulation of the corresponding intensity curve in panel (a). The 3D curve corresponds to the wash-out of this structure in panel (j). The $3 \mathrm{D}$ source function in panel $(\mathrm{h})$ shows a slanted dynamic fibril originating from the magnetic concentration, appearing as a substantial low-reaching green $\eta B^{*}$ contribution, but even this does not affect the last panel because $\tau_{\nu_{0}}=1$ deepens within the concentration. Thus, this 3D simulation confirms the 1D conclusion of Bruls et al. (1992) that the source functions of the $\mathrm{Na}$ I D lines are dominated by resonance scattering.

\subsection{Na I $D_{1}$ Formation in Specific Features}

The first quartet of Figure 9 is a graphical breakdown of $\mathrm{Na}$ I $\mathrm{D}_{1}$ formation for the brightest magnetic concentration marked with a plus sign in Figure 7, using the format developed by Carlsson \& Stein $(1994,1997)$ for $\mathrm{Ca} \mathrm{II} \mathrm{H}_{2 V}$ grains. It rewrites the contribution function to the vertically emergent intensity $C_{I}=d I_{v} / d z=S_{v} \exp \left(-\tau_{v}\right) \chi_{\nu}$, with $\chi_{\nu}$ being the total extinction coefficient, into a product of the three terms $\chi_{v} / \tau_{\nu}$, $S_{v}$, and $\tau_{v} \exp \left(-\tau_{v}\right)$ that are plotted as grayscale quantities in the first three panels with $C_{I}$ as their product in the fourth one. The three terms in this decomposition demonstrate that a large contribution to intensity is made at those positions that have a large opacity at low optical depth, with a high source function around optical depth unity. The line source function in the second panel is frequency independent because of the assumption of complete re-distribution. Strictly speaking one should plot the total source function here. However, to increase readability we avoid the cluttered modulation of the frequency-dependent total source function and plot the line source function instead. This is justified because the line source function becomes equal to the continuum source function in the deep-formed LTE wings and dominates the total source function in the scattering line core.

The emergent $\mathrm{Na}$ I $\mathrm{D}_{1}$ profile in the last panel of the first quartet is very asymmetric. It shows a bright hump at nominal line center that causes the extraordinary brightening of this feature in the second panel of Figure 7. The actual line core is redshifted over $7 \mathrm{~km} \mathrm{~s}^{-1}$. The formation mechanism of this profile is rather similar to the formation of internetwork Ca II $\mathrm{H}_{2 V}$ grains. A large $\left(10 \mathrm{~km} \mathrm{~s}^{-1}\right)$ downdraft in high layers moves the core opacity redward. Its inward integration at the profile minimum reaches $\tau=1$ at $z=0.5 \mathrm{Mm}$, where the source function is dominated by scattering and has uncoupled from the Planck function. The profile minimum is much darker than the emergent intensity at nominal line center. However, it still stands out in the Dopplershift-compensated panels of Figure 7, evidencing a relatively high source function notwithstanding the signature loss by scattering. Figure 7 suggests that the same occurs in other magnetic concentrations.

Several circumstances conspire to produce the bright hump in the profile at nominal line center. Because of the gradient in the downflow velocity there is a location with high $\chi_{v} / \tau_{v}$ at $\Delta v=0$ and $z=0.3 \mathrm{Mm}$ (upper left panel). This location happens to lie close to optical depth unity at that frequency (lower left panel). Most importantly, the source function is not yet completely set by scattering, and partially follows the increase of the Planck function above $z=0.1 \mathrm{Mm}$ (dotted line, upper right panel). This gives rise to the local maximum in the source function in the upper right panel of the first quartet. All effects combined yield a high contribution function (lower right panel) and thus a peak in the profile. There is no corresponding peak at the red side of the profile because $\chi_{\nu} / \tau_{v}$ (upper left panel) is low where $\tau_{v}=1$ coincides with the location of the source function maximum because of the velocity gradient in the atmosphere.

The second quartet of Figure 9 shows a similar breakdown at the asterisk in the cool cloud in the bottom panel of Figure 7. In this case, the profile asymmetry is minor, there are only a small downdraft below $z=0.3 \mathrm{Mm}$ and a small updraft above this height. The Planck function (dotted) drops steeply to $2400 \mathrm{~K}$ 
at $z=1.0 \mathrm{Mm}$. The source function happens to follow it far out, but it actually uncouples already at $z=0.2 \mathrm{Mm}$ where scattering sets in. It levels out at a radiation temperature $3100 \mathrm{~K}$ near $z=0.6 \mathrm{Mm}$ where $\tau_{\nu_{0}}=1$. The resulting line core is very dark.

The third quartet of Figure 9 shows another breakdown for the slender hot front marked with a rhombus in the bottom panel of Figure 7, where $\mathrm{Na} \mathrm{I}_{1}$ has maximum formation height with $\tau_{v_{0}}=1$ at $z=1.1 \mathrm{Mm}$. The Planck function has an extended minimum for $T \approx 5000 \mathrm{~K}$ and then rises steeply with height, through $6000 \mathrm{~K}$ at $z=1.1 \mathrm{Mm}$, but this is not followed by the source function which uncouples much deeper and has a radiation temperature of $3400 \mathrm{~K}$ at $z=1.1 \mathrm{Mm}$. Thus, although $\mathrm{Na}_{\mathrm{I}} \mathrm{D}_{1}$ is formed very high at this location, it does not show the relatively high temperature there. Note the large difference in line-core formation height between the cool cloud of the second quartet and the hot front of third quartet. This is caused by the strong dependence of the $\mathrm{Na} \mathrm{I}_{1}$ opacity on the electron density. The cool cloud has a low temperature, hence a low LTE electron density and thus a low opacity due to relative lack of photorecombination (see Section 3.4). The hot front has a relatively high temperature throughout the whole column, so that the relatively high opacity results in a large formation height.

For comparison, the fourth quartet shows the corresponding Ca II $8542 \AA$ formation breakdown at the location of the hot front, confirming that this line is formed higher and has better coupling to the temperature: this location is quite bright in Ca II $8542 \AA$ (Figures 3 and 4 of Paper I).

\section{DISCUSSION}

\subsection{LTE Ionization versus Non-equilibrium Ionization}

The MHD simulation is quite realistic in the photosphere but arguably less so higher up. One obvious imperfection is the assumption of LTE Saha-Boltzmann partitioning for hydrogen and the electron-donor metals. These are largely neutral in the simulation's cool clouds, but sodium gets largely ionized there in our subsequent NLTE statistical-equilibrium modeling for that element because its ionization is dominated by hot photospheric radiation. The same would be the case for the other electron donors in such modeling. Worse, Carlsson \& Stein (2002) and Leenaarts et al. (2007) have shown that for hydrogen the assumption of statistical equilibrium fails also, requiring the evaluation of non-equilibrium ionization-recombination balancing in which the past history of a given gas parcel affects its state, in particular suffering slow recombination in the cool aftermath of a fast-ionizing hot shock passage. Similar slowness may affect other electron-donor elements, although hydrogen is likely the worst. Therefore, the low electron densities in the cool clouds in Figure 5, with their large effect on the $\mathrm{Na}$ I $\mathrm{D}_{1}$ $\tau=1$ height in the bottom panel of Figure 7, are likely artifacts. The actual electron density may remain as high as $N_{e} / N_{\mathrm{H}} \approx 10^{-2}$ in shocked gas, causing up to $10^{4}$ increase in $\mathrm{H}^{-}$opacity and therefore increased radiative heating above the photosphere wherever the $\mathrm{H}^{-}$bound-free continuum has $\overline{J_{v}}>B_{v}$. So perhaps the cool clouds will not become so cool in the case of non-equilibrium ionization. On the other hand, slow balancing affects the energy budget intrinsically by not instantaneously converting thermal energy into ionization or releasing it in recombination, resulting in larger temperature contrast across shocks (Carlsson \& Stein 1992) and longer-lived cooler post-shock temperatures (Leenaarts et al. 2007). It is hard to predict what cool clouds future simulations with improved ionization treatment will produce, but probably these will have larger electron density than the cool clouds here, and possibly larger $\mathrm{Na}$ I $\mathrm{D}_{1}$ opacity.

\subsection{Chromosphere or Clapotisphere}

Since we use only a single snapshot of the simulation here, we cannot identify the nature of its fine structure in higher layers, that would require analysis of its temporal evolution. Nevertheless, comparison with the 2D simulation of Leenaarts et al. (2007) and the 3D simulation of Martínez-Sykora et al. (2009) suggests that many of the fine-scale features in Figures 58 are due to shock dynamics, with slanted wave guiding along field lines in the neighborhood of the magnetic concentrations producing short dynamic fibrils (see Hansteen et al. 2006; De Pontieu et al. 2007; Paper I). The scene in the rightmost column of Figure 5 consists of slender hot shock fronts and cool expansion clouds. There appear to be no long fibrils in the simulation that would be comparable to those typically observed near network in $\mathrm{H} \alpha$ and in Ca II $8542 \AA$ (Figure 1). This is consistent with the observed $\mathrm{Na}$ I $\mathrm{D}_{1}$ profile minimum intensity image shown in Figure 1 which also shows almost no evidence of fibrils in this quiet region.

The simulated Ca II $8542 \AA$ line-center image from the same snapshot in Figure 3 of Paper I does not contain such long fibrils either and shows larger disparity with the observed line-center scene than occurs here between panel (e) of Figure 3 and panel (d) of Figure 7. We therefore conclude that the simulation does not contain the dense carpets of extended fibrils that constitute the magnetically dominated chromosphere, but that it models what we call the clapotisphere quite well. The simulation is a fair description of what is observed in $\mathrm{Na}_{\mathrm{I}} \mathrm{D}_{1}$ but is deficient in H $\alpha$-type fibrils in Ca II $8542 \AA$. Such deficiency does not really matter for $\mathrm{Na}_{\mathrm{I}} \mathrm{D}_{1}$ because its intensity is determined well below the clapotispheric regime anyhow: although its $\tau_{\nu_{0}}=1$ height in the third quartet of Figure 9 is clapotispheric, the source function there is dominated by $\bar{J}$ photons originating much deeper. Only the core Dopplershift is set at clapotispheric heights, but only outside the transparent cool clouds.

\subsection{Magnetic Concentrations in Na I $D_{1}$ and Ca II $8542 \AA$}

The magnetic concentrations appear bright in both the observations and the simulation, even after compensation for the Doppler-shift imposed by the large downdrafts that tend to occur at their locations, also both in the observations and the simulation. In the simulation, this brightness is due to higher-thanelsewhere temperatures in the upper photosphere $(z=0.3 \mathrm{Mm}$ temperature panel of Figure 5).

Near and within magnetic concentrations, field-guided shocks tend to appear deeper than elsewhere, as was already shown in the classical 2D simulation of Steiner et al. (1998) and confirmed later by the dynamic fibril studies of Hansteen et al. (2006) and De Pontieu et al. (2007). The strong narrow downdrafts in most magnetic concentrations (bottom row of Figure 5) may be postshock downdrafts. As diagnosed in the first quartet of Figure 9, they cause magnetic-concentration brightening with a similar formation mechanism as the asymmetric $\mathrm{Ca}$ II $\mathrm{H} \& \mathrm{~K}$ violetwing brightening in non-magnetic $\mathrm{H}_{2 V}$ and $\mathrm{K}_{2 V}$ grains. These sample the sub-canopy shocks in the internetwork at $z \approx 1 \mathrm{Mm}$. $\mathrm{Na}_{\mathrm{I}} \mathrm{D}_{1}$ is formed much lower and samples only the deep-sited shocks in and near magnetic concentrations. The formation of Ca II $8542 \AA$ is intermediate; the large brightness of the magnetic 
concentration in Figure 4 of Paper I is similarly formed through higher-up downdraft.

The breakdown for the magnetic concentration in Figure 9 shows a raised temperature (Planck function in the second panel) over $z=0.3-0.8 \mathrm{Mm}$; it is the hottest location in the $z=0.3 \mathrm{Mm}$ temperature panel of Figure 5. The other magnetic concentrations have similarly raised temperatures at this height. The corresponding source function hump at $z=0.3 \mathrm{Mm}$, which produces the blue-wing peak at nominal line center, lies within the upper photosphere outside, in the reversed granulation regime (Figure 5).

The vertical velocity curve in the first quartet of Figure 9 (overplotted in all panels) displays a series of steps suggesting shock passages. If so, these are less well defined, with less obvious response in temperature, than those in Figures 57 of Carlsson \& Stein (1997) from their non-magnetic $\mathrm{H}_{2 V}$ grain simulation with the 1D RADYN code. That code used an adaptive mesh and had much better vertical resolution than the fixed mesh of OSC. Also, in 3D, shocks are likely not to run up straight even within magnetic concentrations. Analysis of the full time-dependent simulation will tell whether this velocity pattern indeed stems from shocks and may establish whether shock heating matters in setting this deep-seated temperature increase in magnetic concentrations, as suggested by the high correspondence between excess temperature and excess downdraft in magnetic concentrations in the second column of Figure 5.

\subsection{Comparison Between Observation and Simulation}

The observed and simulated scenes in panel (e) of Figure 3 and panel (d) of Figure 7 show similar morphology. Within the simulation, $\mathrm{Na}$ I $\mathrm{D}_{1}$ is primarily a magnetic-concentration mapper in its brightness distribution, sampling these in the upper photosphere and including wide scattered-light aureoles. Elsewhere, the line-center intensities are fully dominated by scattering, producing very dark source function sampling with much wash-out of thermal structure. Since these formation characteristics are primarily photospheric, where we believe our simulation to be realistic, we suggest that the same apply to the Sun.

In the simulation only the core Dopplershifts, imposed at the last scattering, are likely to sample clapotispheric heights, and that only outside the cool clouds where sodium is fully ionized. The Dopplershift map in panel (f) of Figure 3 has similar slender magnetic-concentration downdrafts as in the companion panel (f) of Figure 7 but less other fine structure, in particular missing out on the considerable downflows in cool clouds and the sharp, slender upflow features from hot shocks that the simulation presents. These differences may be attributable to lack of resolution in the observations and/or overestimation of clapotispheric dynamics in the simulation. At this stage, we cannot tell.

\section{CONCLUSIONS}

Based on the analysis of our simulation we conclude that the brightest quiet-Sun features observed in the core of $\mathrm{Na}$ I $\mathrm{D}_{1}$ are also the deepest: magnetic concentrations sampled at $z \approx 0.3-0.5 \mathrm{Mm}$. The lower height holds when one does not compensate for Dopplershifts in the line-center sampling and a core shift causes additional brightening. This happens in particular when the magnetic concentrations harbor higherlayer downdrafts producing blue-wing peaks through the same line-formation mechanism that causes non-magnetic internetwork $\mathrm{H}_{2 V}$ and $\mathrm{K}_{2 V}$ grains in $\mathrm{Ca}$ II $\mathrm{H}$ \& $\mathrm{K}$. Such downdrafts occur in most magnetic concentrations in the simulation and seem to occur regularly also in the observations, although even our high-resolution IBIS imaging barely resolves them.

The next brightest component in quiet-Sun $\mathrm{Na}$ I $\mathrm{D}_{1}$ scenes is relatively bright aureoles surrounding the magnetic concentrations, due to resonance scattering of $\mathrm{Na}$ I $\mathrm{D}$ radiation from the magnetic concentrations. This is very much a 3D phenomenon requiring 3D NLTE modeling, as demonstrated in Figure 8.

Elsewhere, quiet-Sun Na I $D_{1}$ scenes are dark even though at certain locations the formation height may be located much higher up, around $z=1 \mathrm{Mm}$ within hot clapotispheric shock fronts. Their high temperature has no response in the $\mathrm{Na}$ I $\mathrm{D}_{1}$ source function due to the strong resonance scattering in this line, but it does for the Ca II $8542 \AA$ line which combines higher formation with better temperature sensitivity.

The upshot is that in quiet-Sun areas the Na I $\mathrm{D}_{1}$ core displays primarily an upper-photosphere rendering of magnetic concentrations on the solar surface, with roughly the same surface patterning as in unsigned magnetograms or in proxy diagnostics such as G-band and $\mathrm{H} \alpha$-wing bright points, but contaminated with brightening from downdrafts and 3D-scattered aureoles. This similarity explains the correlation between photospheric magnetic flux density and $\mathrm{Na}$ I $\mathrm{D}_{1}$ core brightness reported by Cauzzi et al. (2000), and it makes $\mathrm{Na}_{\mathrm{I}} \mathrm{D}_{1}$ just another photospheric-field indicator in irradiance monitoring. It is not a chromosphere diagnostic, not even a useful clapotisphere one. Only usage of information imposed at the last scattering (Dopplershift, polarization) seems of interest, but that suffers from the large variation in the height of the $\tau=1$ surface at line center.

The magnetic concentrations in the simulation are hotter at the $z=0.3-0.5 \mathrm{Mm} \mathrm{Na}$ I $\mathrm{D}_{1}$ line-center sampling height than the outside upper photosphere with reversed granulation. Further analysis of the full time-dependent simulation may clarify how this heating comes about; $\mathrm{Na}$ I $\mathrm{D}_{1}$ may be useful to constrain the mechanism.

J.L. acknowledges financial support by the European Commission through the SOLAIRE Network (MTRN-CT-2006035484). R.J.R. acknowledges travel support from the USOSP International School for Solar Physics funded by the European Commission (MEST-CT-2005-020395). This research was supported by the Research Council of Norway through grant 170935/V30 and through grants of computing time from the Programme for Supercomputing. The IBIS observing relied on DST observers; M. Bradford, J. Elrod, and D. Gilliam. We also thank Ryoko Ishikawa for her collaboration in obtaining the Hinode and IBIS observations. IBIS was constructed by INAF/OAA with contributions from the University of Florence, the University of Rome, MIUR, and MAE, and is operated with support of the US National Solar Observatory (NSO). The NSO is operated by the Association of Universities for Research in Astronomy, Inc., under cooperative agreement with the US National Science Foundation. Hinode is a Japanese mission developed and launched by ISAS/JAXA, with NAOJ as domestic partner and NASA and STFC (UK) as international partners. It is operated by these agencies in co-operation with ESA and NSC (Norway). The SOT/SP Level 1D data were generously provided by Bruce Lites. This study benefited from fruitful discussions at and supported by the International Space Sciences 
Institute at Bern, Switzerland, and from NASA's Astrophysics Data System.

\section{REFERENCES}

Al, N., Bendlin, C., \& Kneer, F. 2002, A\&A, 383, 283

Berger, T. E., et al. 2004, A\&A, 428, 613

Botnen, A. 1997, Master thesis, University of Oslo

Bruls, J. H. M. J., Rutten, R. J., \& Shchukina, N. G. 1992, A\&A, 265, 237

Carlson, B. G. 1963, in Methods in Computational Physics, ed. B. Alder, S. Fernbach, \& M. Rotenberg (New York: Academic), 1

Carlsson, M. 1986, Uppsala Astronomical Observatory Reports No. 33

Carlsson, M., \& Stein, R. F. 1992, ApJ, 397, L59

Carlsson, M., \& Stein, R. F. 1994, in Proc. Mini-workshop, Chromospheric Dynamics, ed. M. Carlsson (Oslo: University), 47

Carlsson, M., \& Stein, R. F. 1997, ApJ, 481, 500

Carlsson, M., \& Stein, R. F. 2002, ApJ, 572, 626

Cauzzi, G., Falchi, A., \& Falciani, R. 2000, A\&A, 357, 1093

Cauzzi, G., Reardon, K., Rutten, R. J., Tritschler, A., \& Uitenbroek, H. 2009, A\&A, 503, 577

Cavallini, F. 2006, Sol. Phys., 236, 415

Cheung, M. C. M., Schüssler, M., \& Moreno-Insertis, F. 2007, A\&A, 461, 1163

De Pontieu, B., Hansteen, V. H., Rouppe van der Voort, L., van Noort, M., \& Carlsson, M. 2007, ApJ, 655, 624

Fontenla, J. M., Avrett, E. H., \& Loeser, R. 1993, ApJ, 406, 319

Gustafsson, B. 1973, Uppsala Astron. Obs. Ann., 5, 6

Hansteen, V. H., Carlsson, M., \& Gudiksen, B. 2007, in ASP Conf. Ser. 368, The Physics of Chromospheric Plasmas, ed. P. Heinzel, I. Dorotovič, \& R. J. Rutten (San Francisco, CA: ASP), 107

Hansteen, V. H., De Pontieu, B., Rouppe van der Voort, L., van Noort, M., \& Carlsson, M. 2006, ApJ, 647, L73
Leenaarts, J., \& Carlsson, M. 2010, in Proc. 2nd Hinode Science Meeting, in press

Leenaarts, J., Carlsson, M., Hansteen, V., \& Rouppe van der Voort, L. 2009, ApJ, 694, L128

Leenaarts, J., Carlsson, M., Hansteen, V., \& Rutten, R. J. 2007, A\&A, 473, 625

Leenaarts, J., Rutten, R. J., Carlsson, M., \& Uitenbroek, H. 2006, A\&A, 452, L15

Leenaarts, J., \& Wedemeyer-Böhm, S. 2005, A\&A, 431, 687

Martínez-Sykora, J., Hansteen, V., \& Carlsson, M. 2008, ApJ, 679, 871

Martínez-Sykora, J., Hansteen, V., DePontieu, B., \& Carlsson, M. 2009, ApJ, 701,1569

Neckel, H., \& Labs, D. 1984, Sol. Phys., 90, 205

Nordlund, A. 1982, A\&A, 107, 1

Nordlund, A., Stein, R. F., \& Asplund, M. 2009, Living Rev. Solar Phys., 6, 2

Reardon, K. P., \& Cavallini, F. 2008, A\&A, 481, 897

Reardon, K. P., Uitenbroek, H., \& Cauzzi, G. 2009, A\&A, 500, 1239

Righini, A., Cavallini, F., \& Reardon, K. 2010, A\&A, in press

Rimmele, T. R. 2004, Proc. SPIE, 5490, 34

Rouppe van der Voort, L., et al. 2005, A\&A, 435, 327

Rutten, R. J. 1995, in ESA Special Publication 376, Helioseismology, ed. J. T. Hoeksema et al. (Noordwijk: ESA Publication Divison), 151

Shelyag, S., Schüssler, M., Solanki, S. K., Berdyugina, S. V., \& Vögler, A. 2004, A\&A, 427, 335

Skartlien, R. 2000, ApJ, 536, 465

Solanki, S. K. 1987, PhD thesis, Swiss Federal Institute of Technology

Solanki, S. K. 1993, Space Sci. Rev., 63, 1

Spruit, H. C. 1977, PhD thesis, University of Utrecht

Steiner, O., Grossmann-Doerth, U., Knölker, M., \& Schüssler, M. 1998, ApJ, 495,468

Uitenbroek, H. 1989, A\&A, 213, 360

Uitenbroek, H., \& Bruls, J. H. M. J. 1992, A\&A, 265, 268

Vernazza, J. E., Avrett, E. H., \& Loeser, R. 1981, ApJS, 45, 635

Wedemeyer, S., Freytag, B., Steffen, M., Ludwig, H.-G., \& Holweger, H. 2004, A\&A, 414, 1121 